\title{
IMPORTANCIA DEL REGISTRO NEUROFISIOLÓGICO EVOCADO DURANTE LA COLOCACIÓN DE UN NEUROMODULADOR SACRO.
}

\author{
Federico L. Zeller' y Guillermo Lassalle?2.
}

Servicio de Urología'. Hospital de Área El Bolsón. Río Negro. Argentina.

Servicio de Fisiatría². Hospital Militar Central. Buenos Aires. Argentina.

\begin{abstract}
Resumen.- OBJETIVO: Para la correcta ubicación del electrodo en sacro 3 (S3) durante la cirugía de implante de un neuromodulador, empleamos en la etapa de estimulación transitoria el registro de potenciales evocados con captación a nivel vesicouretral y en la planta del pie (flexor corto del hallux).

MÉTODO: Realizamos esta experiencia en 2 pacientes. Colocamos un electrodo anular montado sobre una sonda Foley $10 \mathrm{~F}$ en el cuello vesical y una aguja coaxial en flexor corto del hallux de cada bóveda plantar. Al estimular la región sacra registramos los potenciales evocados sacro-vesicouretrales y sacro-plantares.
\end{abstract}

RESULTADOS: Al estimular la raíz S3 registramos un potencial evocado vesicouretral de $25.10 \mathrm{mseg}$ en el primer paciente y de $28.52 \mathrm{mseg}$ en el segundo y un potencial evocado sacro-plantar de $20.80 \mathrm{mseg}$
Federico L. Zeller Belgrano, $301 \mathrm{~Pb} \mathrm{~A}$ 8400 Bariloche. Río Negro. (Argentina) fezeller@ciudad.com.ar zellerfederico@gmail.com Trabajo recibido: 23 de mayo 2006 y 22.46 mseg respectivamente. Dichos potenciales no se observan simultáneamente al estimular otras raíces sacras. El registro de estos potenciales antecede a la observación clínica de respuestas de contracción del esfinter anal y del flexor del primer dedo del pie. Cabe destacar que la respuesta evocada a nivel de la unión uretrovesical es conducida a través de fibras del sistema nervioso vegetativo y la respuesta evocada con captación a nivel de la planta del pie viaja a través del sistema somático.

CONCLUSIONES: El registro de los potenciales evocados sacro-vesicouretrales y sacro-plantares durante la colocación de un neuromodulador sacro es una herramienta útil para asegurar la localización en S3 del electrodo sacro.

Palabras clave: Neuromodulación sacra. Estimulación sacra. Incontinencia de apremio. Síndrome urgencia - frecuencia. Potenciales evocados.

Summary.- OBJECTIVES: Sacral neuromodulation is a new treatment option in patients with urge incontinence, urgency-frequency and urinary retention refractory to the pharmacological therapy. The electrophysiological monitoring provides direct and immediate feedback about the function of nerves. We evaluated the utility of the electrophysiological monitoring to assert the correct position of the lead at the third sacral root (S3).

METHOD: We registered the sacral-vesicourethral and sacral plantar evoked potentials in two patients la 55 years old man and a 37 years old woman) during the implant of a sacral neuromodulator IInterstim $\Theta$, Medtronic, MN USA).

RESULTS: We registered vesico-urethral and sacroplantar potentials in response to sacral stimulation. We found 
vesical evoked potentials after the stimulation of S3 and S4 and plantar responses after the stimulation of S2 and S3. The vesico-urethral evoked potentials had a latency of $25.10 \mathrm{mseg}$ in our first case and $28.52 \mathrm{mseg}$ in the second. The sacroplantar evoked potentials had a latency of 20.80 mseg and 22.46 mseg respectively. We observed the evoked potential register during the surgery prior to the visual motor and sensory responses with a lower intensity of stimulation (average $3.5 \mathrm{mAmp}$ ). The only sacral root in which we could find both responses was in S3.

CONCLUSION: The electrophysiological monitoring was helpful in identifying the right position of the lead at the correct sacral root. It avoided the necessity of high stimulation intensity during the procedure.

Keywords: Neuromodulation. Sacral stimulation. Urge incontinence. Urgency-frequency. Evoked potentials.

\section{INTRODUCCIÓN}

Ante el fracaso del tratamiento farmacológico de la incontinencia de apremio, del síndrome urgencia-frecuencia y de la retención urinaria no obstructiva, el empleo de un neuromodulador sacro se ha convertido en los últimos años en una práctica cada vez más frecuente. La raíz sacra que con mayor frecuencia inerva la región pélvica y perineal que interesa para la estimulación sacra es la tercera (S3); sin embargo la neuroanatomía sacra es bastante variable (1-3). Para el correcto implante del electrodo en S3 se realizan pruebas de estimulación intraquirúrgica observando la contracción del esfínter anal y del flexor corto del primer dedo del pie homolateral. También se interroga al paciente durante la cirugía sobre la percepción de estímulos a nivel escrotal o vaginal. En algunos casos, estas pruebas despiertan sensación de dolor, debido a la intensidad del estímulo provocado. Por esa razón y a fin de objetivar las respuestas reflejas exploradas, decidimos realizar el registro de potenciales evocados sacro-vesicouretrales y sacro-plantares durante la estimulación de las raíces sacras. El control intraoperatorio mediante el auxilio de la exploración neurofisiológica permite confirmar la localización correcta del electrodo, sin requerir una estimulación de gran intensidad.

\section{MATERIAL Y MÉTODOS}

Durante la colocación de un neuromodulador sacro, se realizó en dos pacientes (un varón de 55 años y una mujer de 37 años) el registro intraope- ratorio de los potenciales evocados sacro-vesicouretrales y sacro-plantares mediante un electromiógrafo ATI-Delphos ${ }^{\circledR}$ (ATI-Argentina), de dos canales de registro digital. En ambos se implantó un neuromodulador sacro Interstim ${ }^{\circledR}$ (Medtronic, MN USA)

Procedimiento quirúrgico: la colocación del neuromodulador sacro la efectuamos en 2 etapas.

\section{Primera etapa:}

Se coloca al paciente en decúbito ventral y se marcan mediante fibra indeleble, los reparos óseos que servirán de guía para la localización de los forámenes sacros (3). Infiltramos la zona de punción mediante lidocaína al $2 \%$ sin epinefrina, abarcando los planos profundos hasta llegar al periostio sacro. Mediante un trocar de punción se localiza el foramen sacro y se ingresa al mismo. Se retira el mandril del trocar y se introduce el electrodo de estimulación, que se posiciona bajo control radioscópico. Se procede a realizar la estimulación intraquirúrgica y se comprueban las respuestas evocadas. Localizado el foramen S3 se retira el electrodo y se deja el trocar a modo de reparo. Se efectúa anestesia local de los planos adyacentes al sitio de punción y se incide la piel y el tejido celular subcutáneo, cercano al trocar, hasta encontrar la aponeurosis sacra. Se introduce nuevamente el electrodo dentro del trocar y se retira la camisa del mismo. Se efectúan nuevamente las pruebas de estimulación empleando el electrodo y se comprueban las respuestas evocadas (4). Bajo radioscopia se constata la correcta localización del electrodo. Se fija el mismo a la aponeurosis sacra, mediante una mariposa de fijación provista por el fabricante, que se sutura con material no reabsorbible. Se realiza infiltración mediante lidocaína al $2 \% \sin$ epinefrina de la región glútea localizada por debajo de la cresta ilíaca, lugar donde se alojará en la segunda etapa el generador definitivo. Se inciden la piel y los planos subcutáneos hasta la aponeurosis. Se dirige el extremo distal del electrodo hacia la otra incisión, siguiendo un trayecto que se labra bajo el tejido celular subcutáneo. Allí se conecta el extremo distal del electrodo a un prolongador, que se exterioriza mediante contra-abertura a la misma altura, pero en la región glútea superior contralateral. Este extremo se conectará luego al estimulador transitorio externo. Se controla hemostasia de ambas incisiones y se procede al cierre simple de las mismas.

\section{Segunda etapa:}

Si el paciente mejoró su sintomatología en más de un $50 \%$ con respecto al momento previo al implante, se le coloca un generador definitivo. Se anestesia localmente mediante lidocaína al $2 \% \sin$ epinefrina la región donde se dejó alojado durante la primera etapa la conexión del extremo distal del 
electrodo con el prolongador externo. Se incide sobre la incisión anterior y se desconecta el electrodo del prolongador. Se conecta el electrodo al generador definitivo y se labra un bolsillo subcutáneo a ese nivel, dentro del cual se aloja al generador. Se controla la hemostasia y se procede al cierre simple de la incisión.

Procedimientos de estimulación: Antes de colocar al paciente en posición decúbito ventral, se introduce en la vejiga un electrodo anular (Dantec $($ ) montado sobre un catéter Foley 10F. Una vez en posición quirúrgica, se le coloca al paciente en ambas plantas del pie, una aguja coaxial Dantec $® 9013 \mathrm{~L}$ en cada flexor corto del dedo gordo. Durante la estimulación del electrodo para determinar la localización de S3 (primera etapa de la cirugía), se realiza el registro de los potenciales evocados sacro-vesicouretrales y sacro-plantares.

En la segunda etapa de la cirugía se le coloca al paciente sólo el electrodo anular intravesical y al conectar el electrodo al generador definitivo se efectúa el registro de los estímulos a nivel de la vejiga.

Durante el control ambulatorio de los pacientes se les coloca nuevamente el electrodo anular a nivel del cuello vesical y se registran los estímulos provenientes del generador (tanto transitorio como del definitivo).

Para el registro de los potenciales evocados se empleó un electromiógrafo ATI-Delphos, con dos

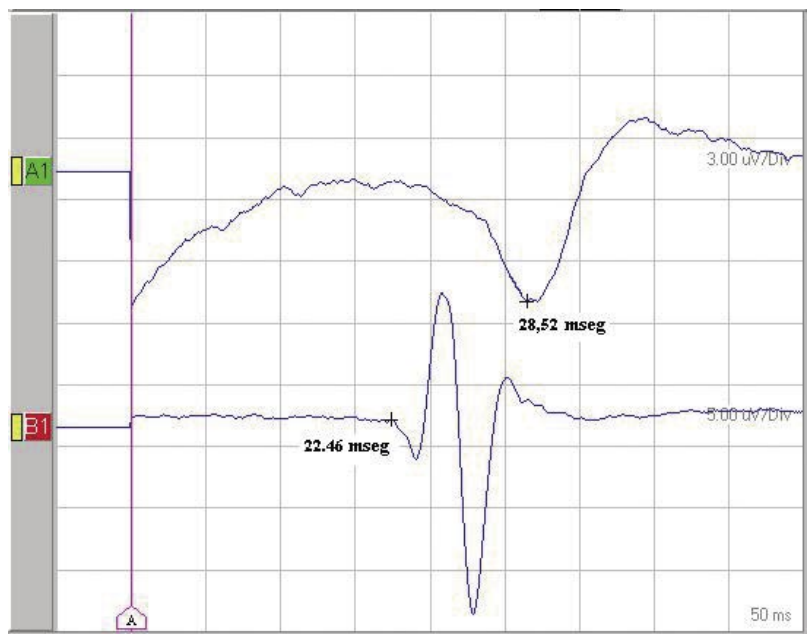

FIGURA 1. Registro intraoperatorio de las respuestas evocadas: El registro superior corresponde a la respuesta evocada vesicouretral ( $1 \mathrm{~cm}=a 3 \mu \mathrm{V} / \mathrm{div}$ ), mientras que el registro inferior corresponde a la respuesta evocada plantar $(1 \mathrm{~cm}=a 5 \mu \mathrm{V} / \mathrm{div})$. canales de registro digital. El estímulo empleado para obtener los potenciales evocados, es una onda cuadrada con una frecuencia de 4.1 pps (pulsos por segundo) y una intensidad promedio de 3,5 mAmp. Los filtros se fijan en un rango entre $1-15 \mathrm{~Hz}$.

\section{RESULTADOS}

Al estimular la región sacra correspondiente a S3, se obtienen respuestas evocadas vesicouretrales y plantares simultáneas. Al estimular sacro 2 se obtienen respuestas plantares, pero no vesicales, mientras que al estimular sacro 4 se obtienen respuestas vesicales, pero no plantares. Las respuestas evocadas vesicouretrales se registraron con una latencia de 25.10 mseg en el primer caso y $28.52 \mathrm{mseg}$ en el segundo (Figura 1). Las respuestas sacro-plantares tuvieron una latencia de $20.80 \mathrm{mseg}$ y $22.46 \mathrm{mseg}$ respectivamente. En ambos casos se estimuló a una intensidad de $3.5 \mathrm{mAmp}$.

Al colocar el electrodo anular, durante el control ambulatorio y en la segunda etapa de la cirugía, se registraron a nivel vesicouretral los estímulos provenientes del generador transitorio y del definitivo respectivamente (Figura 2).

\section{DISCUSIÓN}

El registro de potenciales vesicouretrales mediante el empleo de electrodos anulares fue descrito

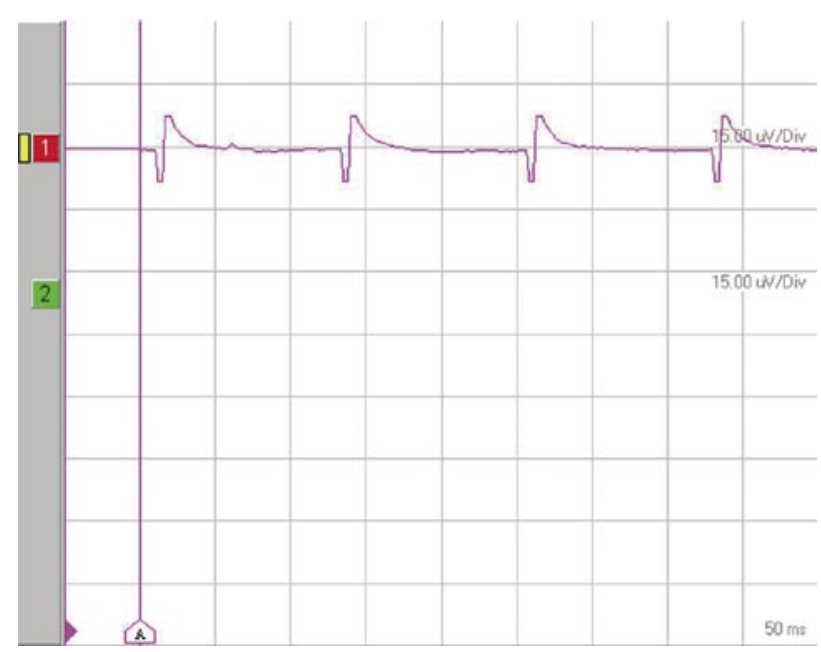

FIGURA 2. Registro vesicouretral ambulatorio de los estímulos provenientes del generador definitivo. 
por Bradley en 1972 (5). Bradley estimulaba a nivel vesical y registraba las respuestas evocadas a nivel del esfínter anal (rango $50-80 \mathrm{mseg}$ ). Hemos empleado este electrodo no para estimular el cuello vesical, sino por el contrario, para registrar las respuestas evocadas a la estimulación de S3, explorando de esa forma solo un segmento del arco reflejo. Estos registros tuvieron una latencia de $25.10 \mathrm{mseg}$ en el primer caso y $28.52 \mathrm{mseg}$ en el segundo. El registro de los potenciales sacro-plantares tuvo un tiempo de latencia menor, a pesar de que la distancia entre el sitio de estimulación y el sitio de captura del estímulo es mayor para éstos (Figura 1). Esta diferencia podría deberse a que las fibras que conducen las respuestas vesicouretrales corresponderían a los nervios pélvicos, fibras autonómicas amielínicas o fibras mielínicas pequeñas, con velocidades de conducción en el rango de las fibras $A$ delta y fibras $C$ o también podría explicarse que por lo general estas respuestas se conducen a través de vías polisinápticas, que podrían ser responsables del mayor tiempo de latencia. En cambio los potenciales evocados sacro-plantares viajarían a lo largo de nervios somáticos mielínicos, con mayor velocidad de conducción.

El beneficio observado durante la estimulación intraquirúrgica reside en la aparición de los potenciales evocados con anterioridad a la aparición de las respuestas visuales percibidas por el examinador o las propioceptivas relatadas por el paciente (contracción del esfínter anal, flexión del dedo gordo del pie o percepción de estimulación a nivel escrotal o vaginal); aún con una intensidad menor de estimulación (3.5 mAmp). Esto puede evitar el dolor durante la estimulación intraquirúrgica, al alcanzar la intensidad necesaria para visualizar la respuesta del esfínter anal (8 a $12 \mathrm{mAmp}$ ). Además el registro de los potenciales evocados vesicouretrales permite asegurar que realmente los estímulos generados en el neuromodulador alcanzan la vejiga.

Por otra parte, el registro de los potenciales evocados vesicouretrales también permite realizar un control adicional del correcto funcionamiento del generador transitorio o definitivo, registrando sus estímulos y corroborando además que la frecuencia de estimulación es la indicada. Este potencial no es una respuesta evocada; se obtiene por transmisión directa debido a la proximidad entre el electrodo estimulador y el electrodo anular. Esto explica porque no podemos registrarlo mediante el electrodo plantar.

\section{CONCLUSIÓN}

El registro neurofisiológico intraoperatorio de las respuestas evocadas permite comprobar la correcta posición del electrodo en S3, con una intensidad menor de estimulación, la cual no provoca dolor al paciente. Permite además corroborar durante los controles ambulatorios la frecuencia de estimulación del generador.

\section{BIBLIOGRAFÍA y LECTURAS RECOMENDADAS ( ${ }^{*}$ lectura de interés $y^{* *}$ lectura fundamental)}

*1. JÜNEMANN, K.P.; LUE, T.F.; SCHMIDT, R.A. y cols.: "Clinical significance of sacral and pudendal nerve anatomy". J. Urol. 139: 74, 1998.

2. BORIRAKCHANYAUAT, S.; ABOSEIF, S.R.; CARROLL, P.R. y cols.: "Continence mechanism of the isolated female urethra: an anatomic study of the intrapelvic somatic nerves". J. Urol. 158: 822, 1997.

3. WARNE, S.A.; GODLEY, M.L.; OWENS, C.M. y cols.: "The validity of sacral ratios to identify sacral abnormalities". BJU 91: 540, 2003.

**4. SIEGEL, S.W.: "Management of voiding dysfunction with an implantable neuroprosthesis". Urol. Clin. N Am 19: 163, 1992.

*5. BRADLEY, W.E.: "Urethral electromyelography”. J. Urol. 108: 563, 1972. 